\title{
Percutaneous radiological gastrostomy: a safe and effective method of nutritional tube placement in advanced ALS
}

\author{
A Chiò, R Galletti, C Finocchiaro, D Righi, M A Ruffino, A Calvo, N Di Vito, P Ghiglione, \\ A A Terreni, R Mutani
}

Background: Enteral nutrition may be required in amyotrophic lateral sclerosis (ALS), and is usually achieved by percutaneous endoscopic gastrostomy (PEG). As PEG is not indicated in patients with severe respiratory impairment, an alternative is percutaneous radiological gastrostomy (PRG), involving air insufflation into the stomach under fluoroscopic guidance for tube insertion.

Objective: To evaluate the safety of PRG and its effect on survival and respiratory function in ALS patients with respiratory failure.

Methods: 25 consecutive ALS patients with severe dysphagia and forced vital capacity (FVC) $<50 \%$ underwent PRG affer October 2000. They were compared with 25 consecutive ALS patients with FVC $<50 \%$ who underwent PEG before October 2000. Respiratory function was evaluated before and after the procedure.

Results: The two groups were similar for all relevant characteristics. PRG was successful in all cases, PEG in 23/ 25 . One patient in each group died after the procedure. The mean survival time after the procedure was 204 days in the PRG group and 85 days in the PEG group $(p<0.004)$. Respiratory function decreased more in the PEG group than in the PRG group $(p<0.02)$.

Conclusions: PRG appears to be safer than PEG in ALS patients with moderate or severe respiratory impairment, and is followed by a longer survival.

\footnotetext{
A
} myotrophic lateral sclerosis (ALS) is a neurodegenerative disorder of adult life characterised by a progressive decline of motor functions at spinal and bulbar level. The involvement of bulbar function includes dysarthria and dysphagia, which in turn cause malnutrition, progressive weight loss, and risk of aspiration pneumonia, and may finally contribute to the patient's death.

Enteral nutrition is widely used to treat dysphagia in ALS. ${ }^{12}$ The insertion of the enteral tube has traditionally been achieved by percutaneous endoscopic gastrostomy (PEG). However, this procedure has major limitations, as it is not recommended in patients with moderate or severe respiratory impairment (that is, with a forced vital capacity (FVC) of less than 50\%), ${ }^{3}$ and cannot be done in subjects with masseter spasticity. ${ }^{4}$ It has been proposed that in these patients the use of non-invasive positive pressure ventilation (NIPPV), oxygen support, and conscious sedation may allow endoscopic enteral tube placement. ${ }^{56}$

Recently, a different method of gastric tube insertion has been proposed in ALS patients. ${ }^{7}$ This method, called percutaneous radiological gastrostomy (PRG), involves air insufflation into the stomach under fluoroscopic guidance for tube insertion. ${ }^{8}$
We have undertaken a study on the safety of PRG and its effect on respiratory function, and on survival after the procedure, in a series of consecutive ALS patients with moderate or severe respiratory involvement.

\section{METHODS \\ PRG procedure}

A nasogastric tube was placed shortly before the procedure to allow insufflation of air into the stomach. Local analgesia was used. No sedation was used and no antibiotics were routinely given. Joshine $\mathrm{N}$-butyl-bromide $(5 \mathrm{mg}$ intravenously) was used to reduce gastric motility and to allow adequate gastric distension (thus reducing the risk of puncture of colon, liver, and other organs). With the patient in supine position, fluoroscopy of the abdomen was undertaken, and the position of the nasogastric tube identified. A site for gastric puncture was then chosen, ideally over the body of the stomach, equidistant from the greater and lesser curves. The needle was inserted into the air filled stomach under fluoroscopic guidance. Aspiration of air bubbles into the syringe confirmed the position of the needle within the stomach. A $12 \mathrm{~F}$ polyurethane tube (Wills Oglesby, Wilson Cook Inc, Bloomington, Indiana, USA) was used, and fixed by means of a loop. During the first day after the procedure the patients received only parenteral nutrition. Enteral nutrition was started on the second day. Major and minor complications of the procedure were recorded. ${ }^{9}$

\section{Clinical follow up and respiratory evaluation}

Patients were followed up prospectively, with two monthly clinic visits. They were evaluated for respiratory function 20 to 30 days before and 20 to 30 days after PRG placement. Respiratory evaluation included spirometry (vital capacity (VC) and FVC per cent of predicted), and arterial blood analysis.

\section{Study population}

The study population included all patients affected by definite or probable ALS, with severe dysphagia or weight loss over $10 \%$, or both, with an FVC under $50 \%$, who consecutively underwent PRG from October 2000 to December 2002 in our ALS centre.

\section{Control population}

The control population included all patients with definite or probable ALS, with VC under 50\%, who underwent PEG in our ALS centre because of severe dysphagia or weight loss

Abbreviations: FVC, forced vital capacity; NIPPV, non-invasive positive pressure ventilation; PEG, percutaneous endoscopic gastrostomy; PRG, percutaneous radiological gastrostomy; VC, vital capacity 
over $10 \%$, or both, before October 2000 . Respiratory evaluation was done as described for the PRG patients.

\section{Statistical analysis}

Survival curves were calculated by the Kaplan-Meier method and compared by log-rank test. Multivariate analysis was done using Cox's stepwise proportional hazard model. ${ }^{10} \mathrm{~A}$ probability $(p)$ value of $<0.05$ was considered significant.

The study was approved by the local ethics committee. All patients signed their written informed consent.

\section{RESULTS}

\section{Study population}

Twenty five patients (12 men, 13 women; mean (SD) age, 68.9 (9.5) years; range 49 to 81 ) underwent PRG in the study period. The mean FVC\% at the time of PRG was $41(9.3) \%$, range $23 \%$ to $48 \%$. At the time of $P R G$, five of these patients were using intermittent NIPPV for a mean of 5.0 (3.3) hours a day, range 2 to 9 .

\section{Control population}

The control population included 25 patients (13 men, 12 women; mean age 65.1 (10.3) years; range 51 to 75 ) with FVC\% under $50 \%$ (mean 40 (8.3)\%; range 33\% to $49 \%$ ) who underwent PEG before October 2000. These patients did not differ significantly from those who underwent PRG for any relevant demographic or prognostic factor (table 1). One patient in each group had severe pseudobulbar palsy.

\section{Safety analysis}

PEG was unsuccessful in two patients $(8 \%)$, in both cases because the stomach was positioned high behind the ribs. No PRG was unsuccessful. One patient from each group died within one month after the procedure. The patient who died in the PRG group was a 74 old woman with an FVC\% of $38 \%$, who was still using NIPPV with a nasal mask. She died seven days after the procedure from a pulmonary embolus secondary to deep venous thrombosis. The patient who died in the PEG group was a 44 year old man with an FVC\% of $45 \%$. He died three days after the procedure from respiratory failure, probably precipitated by the PEG. There were no other major or minor acute complications in either group.

\section{Long term complications}

One patient of the PRG group had a displacement of the tube three months after PRG; however, the tube was easily reinserted without the need for a new intervention. One patient in the PEG group had a rupture of the tube nine months after the procedure, and the tube was substituted at a new intervention. No tube replacement was necessary in either group.

\section{Respiratory function}

Respiratory evaluation one month after PRG showed a mean (SD) decrease of $4.3(2.1) \%$ in the FVC compared with the

\begin{tabular}{llll} 
Table 1 & \multicolumn{3}{c}{ Characteristics of patients and controls } \\
\hline & PRG group & PEG group & p Value \\
\hline Age (years) (mean (SD)) & $68.9(9.5)$ & $65.1(10.3)$ & NS \\
Sex (\% men) & 48 & 52 & NS \\
Type of onset (\% bulbar) & 56 & 52 & NS \\
FVC (\%) (mean (SD))* & $41.4(9.3)$ & $40.9(8.3)$ & NS \\
NIPPV (\%)t & 20 & 8 & NS \\
\hline
\end{tabular}

*Two to three weeks before the procedure. †At the time of the procedure.

FVC, forced vital capacity; NIPPV, non-invasive positive pressure ventilation; PEG, percutaneous endoscopic gastrostomy; PRG percutaneous radiological gastrostomy. evaluation done in the month preceding the procedure. The equivalent value in the PEG group was $8.6(3.4) \%$. The decrease in the PRG group was significantly less than the decrease in the PEG group $(\mathrm{p}<0.02)$.

\section{Survival}

The median survival after gastrostomy was 204 (15) days in the PRG group, and 85 (12) days in the PEG group (fig 1) $(p=0.004)$. In multivariate analysis including NIPPV, the method of gastrostomy was independently related to survival (table 2).

\section{DISCUSSION}

Since the first description in $1994,{ }^{11}$ the use of enteral nutrition in ALS patients with severe dysphagia has become a routine procedure and its effects on undernutrition are well established. ${ }^{4}{ }^{12}$ However, evidence based reviews found major limitations to the use of PEG for enteral tube insertion when the patient has moderate (FVC $30-50 \%$ ) or severe respiratory impairment (FVC <30\%), ${ }^{13}{ }^{14}$ although it is often done in those circumstances. The reasons for this are not entirely clear but may be related to a further reduction in diaphragm mobility during and after the surgical procedure. ${ }^{11}$

To overcome this limitation, an approach based on the use of non-invasive positive pressure ventilation (NIPPV) for ventilatory support with conscious sedation during the surgical procedure has been proposed. ${ }^{56}$ This approach is based on the view that NIPPV improves chronic hypercapnia and normalises nocturnal oxygen saturation in patients with chronic ventilatory insufficiency. However, in both the above series most of the patients were already successfully using NIPPV at the time of the procedure and were thoroughly accustomed to the mask and the ventilatory device. The need for the patient to adapt to NIPPV is a major limitation of this approach, as NIPPV needs prolonged training and is not well tolerated, or is even declined, by about half the patients who try it. ${ }^{14} 15$

Our data suggest that gastrostomy tube placement using PRG is a safe and effective alternative to PEG in patients with moderate or severe respiratory failure, even without the concomitant use of NIPPV. The median survival time after the procedure is in line with the findings of other studies with comparable populations, ${ }^{6}$ and longer than that observed in patients with moderate or severe respiratory failure treated with PEG. ${ }^{411}{ }^{12}$ The success rate of the procedure is very high, confirming the data of other series of patients with $\mathrm{ALS}^{7}$ or other disorders. ${ }^{8}$ Moreover, PRG may also be undertaken in patients in whom PEG has been unsuccessful. ${ }^{7}$

In the present series PRG was followed by a smaller reduction in $\mathrm{FVC} \%$ in the first month after the procedure than was found with PEG. The reason for this is not clear but may be related to the minor nature of the procedure, the shorter duration of the intervention, and the fact that

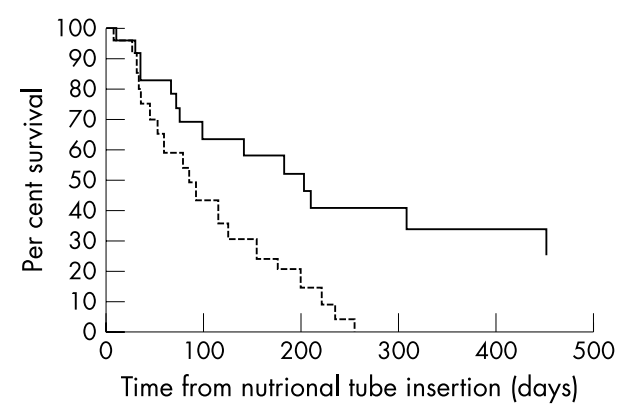

Figure 1 Survival curves of the PRG group (continuous line) and PEG group (dotted line). The difference in survival is significant $(p=0.004)$. 


\begin{tabular}{|c|c|c|c|c|}
\hline Variable & & OR & $95 \% \mathrm{Cl}$ & p Value \\
\hline FVC (\%) & $\begin{array}{l}30 \text { to } 49 \\
<30\end{array}$ & $\begin{array}{l}1 \\
2.87\end{array}$ & 1.35 to 3.18 & 0.001 \\
\hline Intervention & $\begin{array}{l}\text { PRG } \\
\text { PEG }\end{array}$ & $\begin{array}{l}1 \\
1.58\end{array}$ & & 0.03 \\
\hline
\end{tabular}

sedation during the procedure is not required. Moreover, the use of a nasogastric tube in the PRG procedure is an advantage in patients with masseter spasm, who cannot undergo gastroscopy because of the impossibility of opening their mouth. ${ }^{4}$

One disadvantage of PRG is the smaller calibre of the tube, which thus has a greater risk of obstruction. However, the first tube can easily be replaced with one of greater calibre after 15 to 30 days. Another possible problem is the fixation of the tube with a loop, with a greater risk of displacement. However, in our series we observed this event in only one patient, and the tube was easily repositioned.

Further studies, possibly with a controlled comparison of the two methods, are needed to fully define the role of PRG in the treatment of dysphagia in ALS patients before the procedure is included in ALS practice guidelines.

\section{Authors' affiliations}

A Chiò, A Calvo, N Di Vito, P Ghiglione, A A Terreni, R Mutani, Second Division of Neurology, Department of Neuroscience, University of Turin, Italy

D Righi, M A Ruffino, Institute of Diagnostic and Interventional Radiology, University of Turin

R Galletti, C Finocchiaro, Division of Clinical Nutrition, San Giovanni Hospital of Turin

Competing interests: none declared

Correspondence to: Dr Adriano Chiò, Department of Neuroscience, University of Turin, via Cherasco 15, 10126 Torino, Italy; achio@usa.net
Received 9 June 2003

In revised form 14 August 2003

Accepted 23 August 2003

\section{REFERENCES}

1 Bradley WG, Anderson F, Bromberg M, for the ALS CARE Study Group, et al. Current management of ALS: comparison of the ALS CARE database and the AAN practice parameters. The American Academy of Neurology. Neurology 2001;57:500-4

2 Borasio GD, Shaw PJ, Hardiman O, et al. European ALS Study Group. Standards of palliative care for patients with amyotrophic lateral sclerosis: results of a European survey, Amyotrophic Lateral Sclerosis and Other Motor Neuron Disorders 2001;2:159-64.

3 Miller RG, Rosenberg JA, Gelinas DF, et al. Practice parameters: the care of the patient of amyotrophic lateral sclerosis (an evidence-based review): report of the quality Standards Subcommittee of the American Academy of Neurology: ALS Practice Parameters Task Force. Neurology 1999:52:1311-23.

4 Mazzini L, Corra I, Zaccala M, et al. Percutaneous endoscopic gastrostomy and enteral nutrition in amyotrophic lateral sclerosis. J Neurol 1995;242:695-8.

5 Boitano L, Jordan T, Benditt JO. Noninvasive ventilation allows gastrostomy tube placement in patients with advanced ALS. Neurology 2001;56:413-14

6 Gregory S, Siderowf A, Golaszewski AL, et al. Gastrostomy insertion in ALS patients with low vital capacity: respiratory support and survival. Neurology 2002;58:485-7.

7 Thorton FJ, Fotheringham T, Alexander M et al. Amyotrophic lateral sclerosis: enteral nutrition provision - endoscopic or radiologic gastrostomy? Radiology 2002;224:713-17.

8 Barkmeier JM, Trerotola SO, Wiebke EA, et al. Percutaneous radiologic, surgical endoscopic and percutaneous endoscopic gastrostomy/ gastrojejunostomy: comparative study and cost analysis. Cardiovasc Intervent gastrojejunostomy: comparr

9 Shellito PC, Malt RA. Tube gastrostomy techniques and complications. Ann Surg 1985;201:180-95.

10 Parmar MKB, Machin D. Survival analysis. A practical approach. Chichester: John Wiley and Sons, 1995.

11 Mathus-Vliegen LMH, Louwersee LS, Merkus MP, et al. Percutaneous endoscopic gastrostomy in patients with amyotrophic lateral sclerosis and impaired pulmonary function. Gastrointest Endosc 1994;40:463-9.

12 Chiò A, Finocchiaro E, Meineri P, et al. The ALS Percutaneous Endoscopic Gastrostomy Study Group. Safety and factors related to survival after percutaneous endoscopic gastrostomy in ALS. Neurology 1999;53:1123-5.

13 Miller RG. Examining the evidence about treatment in ALS/MND. ALS $2001 ; 2: 3-7$.

14 Kleopa KA, Sherman M, Neal B, et al. Bipap improves survival and rate of pulmonary function decline in patients with ALS. J Neurol Sci 1999;164:82-8.

15 Aboussouan LS, Kham SU, Meeker DP, et al. Effect of noninvasive positivepressure ventilation on survival in amyotrophic lateral sclerosis. Ann Intern Med 1997; 127:450-3. 